\title{
Respiratory Signal Derived from the Smartphone Built-in Accelerometer during a Respiratory Load Protocol
}

\author{
Luis Estrada, Member, IEEE, Abel Torres, Member, IEEE, Leonardo Sarlabous, and \\ Raimon Jané, Senior Member, IEEE
}

\begin{abstract}
The scope of our work focuses on investigating the potential use of the built-in accelerometer of the smartphones for the recording of the respiratory activity and deriving the respiratory rate. Five healthy subjects performed an inspiratory load protocol. The excursion of the right chest was recorded using the built-in triaxial accelerometer of a smartphone along the $x, y$ and $z$ axes and with an external uniaxial accelerometer. Simultaneously, the respiratory airflow and the inspiratory mouth pressure were recorded, as reference respiratory signals. The chest acceleration signal recorded in the $z$ axis with the smartphone was denoised using a scheme based on the ensemble empirical mode decomposition, a noise data assisted method which decomposes nonstationary and nonlinear signals into intrinsic mode functions. To distinguish noisy oscillatory modes from the relevant modes we use the detrended fluctuation analysis. We reported a very strong correlation between the acceleration of the $z$ axis of the smartphone and the reference accelerometer across the inspiratory load protocol (from 0.80 to 0.97 ). Furthermore, the evaluation of the respiratory rate showed a very strong correlation (0.98). A good agreement was observed between the respiratory rate estimated with the chest acceleration signal from the $z$ axis of the smartphone and with the respiratory airflow signal: Bland-Altman limits of agreement between -1.44 and 1.46 breaths per minute with a mean bias of $\mathbf{- 0 . 0 1}$ breaths per minute. This preliminary study provides a valuable insight into the use of the smartphone and its built-in accelerometer for respiratory monitoring.
\end{abstract}

\section{INTRODUCTION}

The widespread use of mobile technologies represents an opportunity to facilitate the clinical practice and improve the healthcare delivery. Mobile health (mHealth), a term referred to the use of modern smart mobile devices (such as smartphones and tablets) in the medical and public health practice has been recognized as important due to its huge potential for bringing solutions in medical services [1].

The first author was supported by the Instituto para la Formación y Aprovechamiento de Recursos Humanos and Secretaría Nacional de Ciencia, Tecnología e Innovación (IFARHU-SENACYT Program) from the Panama Government under grant 270-2012-273. This work was supported in part by the Secretaria d'Universitats i Recerca del Departament d'Economia i Coneixement de la Generalitat de Catalunya (Consolidated research group GRC: 2014 SGR 1569).

L. Estrada, A. Torres, and R. Jané are with the Universitat Politècnica de Catalunya (UPC), the Institut de Bioenginyeria de Catalunya (IBEC) and the Biomedical Research Networking Center in Bioengineering, Biomaterials and Nanomedicine (CIBER-BBN), c/. Baldiri Reixac 4, 08028, Barcelona, Spain. (e-mail: lestrada@ibecbarcelona.eu,abel.torres@upc.edu, rjane@ibecbarcelona.eu).

A. Torres is also with the Escola Universitària d'Enginyeria Tècnica Industrial de Barcelona (EUETIB), Barcelona, Spain.

L. Sarlabous is with the CIBER-BBN and the IBEC (e-mail: lsarlabous@ibecbarcelona.eu).
Moreover, special attention has been paid to using these mobile wireless technologies to improve the live condition of elderly population and individuals suffering from chronic diseases [2]. Respiratory rate (RR), an important vital sign, reflects the respiratory status of an individual and consequently is an indicator of deteriorating health. However, to date, RR is considered the vital sign recorded less frequently by medical staff [3]. To handle this situation, several authors are evaluating novel technologies including the use of smartphones, devices that integrate miniaturized sensors capable of non-invasively collect physiological signals [4], [5]. Additionally, RR has been evaluated by several monitoring technologies, classified in contact and non-contact devices with the patients [6]. Unfortunately, most of these technologies are expensive or are not available for most people. With the penetration of mobile technologies in the market, several strategies are being explored for the recoding of vital signs as the RR. Indeed, previous studies have shown the possibility of using the smartphone built-in sensors such as accelerometers [4], [5] or accessory hardware, as electrocardiogram electrodes or external accelerometers [7] to measure the respiration and derive the RR. In this work, we addressed the use of the built-in accelerometer of the smartphone as a low-cost and an easyto-use alternative for the recording of the chest movement during a respiratory protocol. Secondly, we aim to derive the RR from respiratory activity and compare it to RR extracted from the airflow signal in healthy subjects performing a respiratory protocol based on increments in the inspiratory load.

\section{MATERIAL AND METHODS}

\section{A. Subjects and respiratory protocol}

Five healthy, non-smoking male subjects with no medical history and free of cardiorespiratory diseases were recruited to participate in the study (mean \pm standard deviation: age $34.80 \pm 4.76$ years, height $1.77 \pm 0.08 \mathrm{~m}$, weight $82 \pm 7.21 \mathrm{~kg}$ ). The study was conducted with the subject's written consent, and with the approval of the Institutional Review Board of the Institute for Bioengineering of Catalonia (IBEC), Barcelona, Spain. Subjects underwent an inspiratory load protocol in which they initially inhaled via a mouthpiece without any obstruction (quiet breathing) followed by an attached handheld inspiratory muscle training device (Threshold IMT, Philips Respironics, Amsterdam, The Netherlands) in sitting position and wearing a disposable nose clip to prevent air from escaping. The threshold IMT device imposes an adjustable inspiratory load which limits air to pass through 


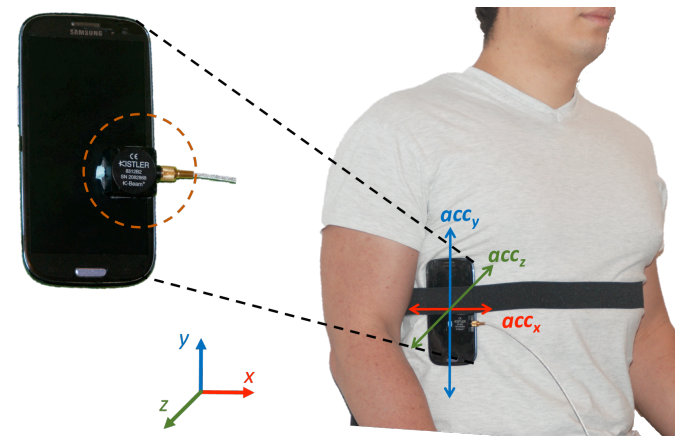

Figure 1. Position of the smartphone and the uniaxial accelerometer placed at the level of the right chest.

the device to the lungs. The expiration remains unloaded. The use of the IMT device was set to $9 \mathrm{cmH}_{2} \mathrm{O}$ and thereafter to 17,25 and $33 \mathrm{cmH}_{2} \mathrm{O}$ of inspiratory load. The inspiratory load protocol without and with the use of the IMT device was performed during 1.5-minutes of work and with 3-minutes of rest. Furthermore, the subjects breathed without any specific breathing pattern.

\section{B. Recording of the signals}

Signals were recorded using the built-in triaxial accelerometer (LMS330DLC, ST Microelectronics) of a smartphone (Samsung Electronics Co., Seoul, South Korea, Galaxy SIII, model GT-I9300) running an Android operating system (version 4.3). This device records the acceleration components $a c c_{x}, a c c_{\mathrm{y}}$ and $a c c_{z}$ along the orthogonal $x, y$ (gravity acceleration) and $z$ directions, respectively, during the breathing motion. The smartphone was securely fastened in a upright position and facing outward to an elastic belt worn at the level of the right chest, between the midaxillary line and the anterior axillary line, and above the costal margin, to the best signal capture, as depicted in Fig. 1. Raw acceleration data was captured employing an Android application (AndroSensor) [8] up to a maximum frequency of $200 \mathrm{~Hz}$. Simultaneously, a reference acceleration signal $\left(\mathrm{A}_{\text {ref }}\right)$, the inspiratory mouth pressure $\left(\mathrm{P}_{\text {mouth }}\right)$ and the respiratory airflow (AF) were recorded. $A_{\text {ref }}$ was recorded using an uniaxial capacitive accelerometer (K-Beam 8305A, Amherst, USA), adhered using double-sided tape to the lower left side of the smartphone (Fig. 1) and coupled with an interface module (UIM100C, Biopac Systems, Inc, Santa Barbara, CA, USA). $\mathrm{P}_{\text {mouth }}$ was measured by a differential pressure transducer (TSD160, Biopac Systems, Inc.). AF was recorded with a pneumotachograph (TSD107B, Biopac Systems). $P_{\text {mouth }}$ and AF signals were amplified using modular differential amplifiers (DAC100C, Biopac Systems, Inc.) with a gain of 50 and an analog low-pass filter with a cut-off frequency of $300 \mathrm{~Hz}$. Data were sampled at a frequency of $2000 \mathrm{~Hz}$ using a data acquisition system (MP100, Biopac Systems, Inc.). Moreover, data were transferred to a personal computer to be displayed in real time on a video screen, allowing us to monitor the breathing and stored to further be off-line processed using the associated software (AcqKnowledge software v.3.2, Biopac Systems, Inc.). Synchronization between equipments was carried out manually. Afterwards, $\mathrm{P}_{\text {mouth }}, \mathrm{AF}$ and $\mathrm{A}_{\text {ref }}$ signals were subsampled to a frequency of $100 \mathrm{~Hz}$. AF signal was digitally band-pass filtered using a zero-phase second-order Butterworth filter with a cut frequency of 0.05 and $1 \mathrm{~Hz}$. All

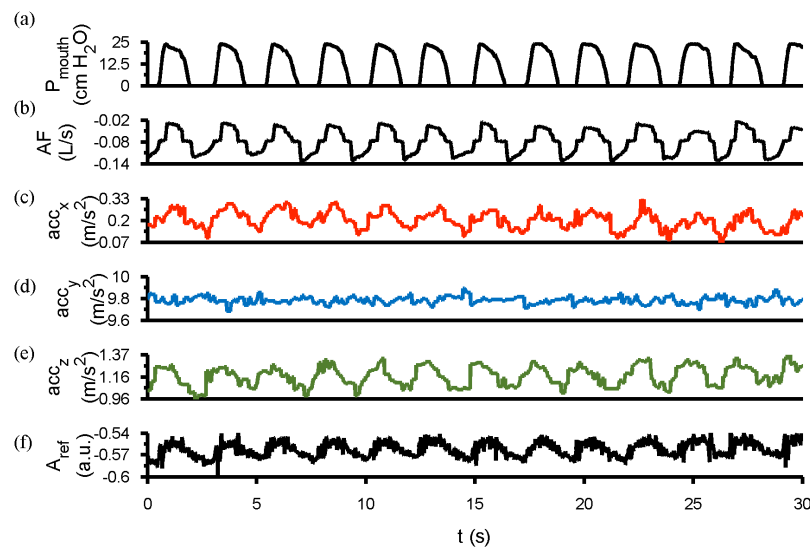

Figure 2. Example of the raw signals acquired during the inspiratory load protocol with a load of $25 \mathrm{cmH}_{2} \mathrm{O}$. (a) Inspiratory pressure $\left(\mathrm{P}_{\text {mouth }}\right)$, (b) Airflow (AF). (c), (d) and (e) correspond to the accelerometer signals of the smartphone $a c c_{x}, a c c_{y}, a c c_{z}$, respectively, and (f) is the accelerometer reference signal $\left(\mathrm{A}_{\mathrm{ref}}\right)$.

data was analyzed offline using MATLAB (v. R2011b, Natick, MA, USA). Fig. 2 shows an example of the $\mathrm{P}_{\text {mouth, }}$ $\mathrm{AF}$ and accelerometer signals.

\section{Preprocessing of the smartphone accelerometer signal}

The sampling frequency of the smartphone is nonconstant. Taking the above into consideration, we first estimated the sampling frequency along all the accelerometer data, being found in the range of $199.99 \pm 105.00 \mathrm{~Hz}$ (mean \pm standard deviation). Subsequently, the acceleration data of the smartphone were resampled to a fixed frequency of 100 $\mathrm{Hz}$ using interpolation. We have chosen the $a c c_{z}$ signal as the most representative component of the acceleration, although, some of the respiratory activity was also recorded in the $a c c_{x}$ and the $a c c_{y}$ components of the acceleration.

\section{Denoising of accelerometer signals with the Ensemble Empirical mode decomposition}

Due to its nature, the accelerometer signals of the smartphone can be denoised by applying the empirical mode decomposition (EMD), an adaptive technique suitable for non-linear and non-stationary data analysis [9]. EMD decomposes a signal into a sum of zero-mean oscillating components referred to as intrinsic mode functions (IMFs). These IMFs are obtained in decreasing order of frequency, with the first IMFs containing high frequency components (related to the muscle vibratory activity) and the last IMFs containing the low frequency components (more associated to the breathing activity) [10]. However, a major drawback of the EMD is the mode-mixing phenomenon, that is, a single IMF either including signals of dramatically disparate scales, or a signal of the same scale appearing at different IMFs, and thus, with the consequence of losing its physical interpretation [11]. To overcome this undesired effect in the original EMD, the Ensemble Empirical Mode Decomposition (EEMD), a noise data assisted method, was developed [11]. In EEMD, white Gaussian noise is added to the original signal and then it is decomposed by the classical EMD algorithm into IMFs. This process is repeated for a certain number of trials. The added noise is suppressed in the ensemble averaged of the corresponding IMFs. We generated 100 noisy trials and the Gaussian noise was added with a 
TABLE I. CORRELATION COEFFICIENTS BETWEEN THE RAW A AND THE $\mathrm{ACC}_{z}$ SIGNALS AT DIFFERENT LEVELS OF INSPIRATORY LOAD

\begin{tabular}{|c|c|c|c|c|c|}
\hline Subject & $\mathbf{Q B}$ & $\mathbf{9} \mathbf{~} \mathbf{m}_{\mathbf{2}} \mathbf{O}$ & $\mathbf{1 7} \mathbf{~} \mathbf{m}_{\mathbf{2}} \mathbf{O}$ & $\mathbf{2 5} \mathbf{~} \mathbf{m H}_{\mathbf{2}} \mathbf{O}$ & $\mathbf{3 3} \mathbf{~ m ~}_{\mathbf{2}} \mathbf{O}$ \\
\hline 1 & 0.96 & 0.96 & 0.96 & 0.93 & 0.88 \\
\hline 2 & 0.88 & 0.88 & 0.93 & 0.93 & 0.92 \\
\hline 3 & 0.96 & 0.96 & 0.97 & 0.96 & 0.96 \\
\hline 4 & 0.87 & 0.94 & 0.93 & 0.90 & 0.94 \\
\hline 5 & 0.83 & 0.87 & 0.80 & 0.81 & 0.82 \\
\hline mean \pm SD & $0.90 \pm 0.06$ & $0.92 \pm 0.04$ & $0.92 \pm 0.07$ & $0.91 \pm 0.06$ & $0.91 \pm 0.06$ \\
\hline
\end{tabular}

QB: quiet breathing, SD: standard deviation.

signal to noise ratio of $30 \mathrm{~dB}$. The added noise was low-pass filtered using a zero-phase eight-order Butterworth filter with a cut frequency of $40 \mathrm{~Hz}$. The main challenge when the EMD-based methods are used as denoising techniques is to adopt a criterion for the adequate selection of those IMFs with relevant information and reject the noise. The EMD was calculated using the routine developed in [12]. On the other hand, in [13] it has been suggested to use the Detrended Fluctuation Analysis (DFA) as a metric to identify noisy IMFs from EMD (DFA-EMD denoising). In this work, we extended the use of DFA to EEMD. DFA is a scaling analysis method for the study of long-range correlations in nonstationary time series data under the presence of noise [14]. Briefly, DFA first integrates the time series after mean subtraction. Then the integrated time series is split into equally sized boxes. In each box, a least-squares line is fitted to the data, which represents the local trend in the box. We calculate the detrended fluctuation function by subtracting the integrated time series and the local trend in each box. The root mean square (RMS) of the detrended fluctuation function is calculated. This procedure was repeated for different box sizes to provide a relationship between the average fluctuations and the box size. We use a box size ranging from 100 to 1000 in equals steps of 100. Finally, to reveal the presence of self-similarity scale in the time series, we estimate the scaling exponent $\alpha$ evaluating the slope of the RMS detrended fluctuation function and box sizes in a $\log -\log$ plot. The correlations of the time series, characterized by the scaling exponent $\alpha$, can be interpret as follows: $\alpha<$ 0.5 is anti-correlated, $\alpha \cong 0.5$ is uncorrelated or white noise, $0.5<\alpha<1$ is correlated, $\alpha \cong 1$, is pink noise, $1<\alpha<1.5$ is non-stationary or random walk, $\alpha \cong 1.5$ is Brownian noise. The $\alpha$ exponent was calculated for each IMF in order to distinguish between noise and noise free IMFs. Those IMFs whose $\alpha$ value was greater than or equal to 0.5 (correlated data) were related to the respiration while the rest of IMFs
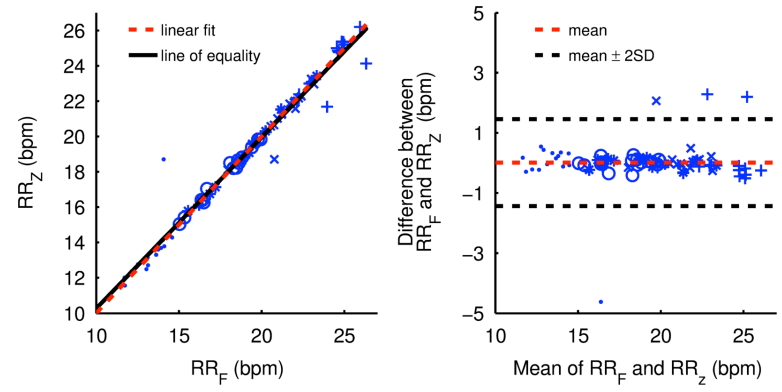

Figure 3. Comparison between the mean $R_{F}$ and $R R_{Z}$ values. (a) Correlation plot and (b) Bland-Altman plot statistics. Each marker represents the measurement in an individual subject each 30 -sec across the inspiratory load protocol. bpm: breaths per minute. were considered as noise. The last mode (residue) of the EEMD was excluded from the study. Afterward, the accelerometer signal was reconstructed by summing the IMF identified with respiratory information.

\section{E. Correlation between the reference and the smartphone accelerometer signal}

Pearson's correlation coefficient was used to analyze the linear association between the $\mathrm{A}_{\text {ref }}$ and the $a c c_{z}$ raw signals across the inspiratory load levels.

\section{$F$. Derived respiratory rate and analysis}

To estimate the RR, first, the maximum values of the respiratory signal ( $\mathrm{R}$ point) were detected in each respiratory cycle. Then, every 30 -sec the RR was calculated as the average of the differences between the successive $\mathrm{R}$ points. This procedure was carried out in the $\mathrm{AF}\left(\mathrm{RR}_{\mathrm{F}}\right)$ and $a c c_{z}$ $\left(R R_{Z}\right)$ signals. Strength and agreement between the $R R_{F}$ and the $\mathrm{RR}_{\mathrm{Z}}$ were determined by the Pearson's correlation and the Bland-Altman plot, respectively. Moreover, the error was measured by the root mean square error (RMSE) over pairs of RRs at each level of inspiratory load.

\section{RESULTS}

The RR values for all subjects ranged from 11.57 to 26.32 breaths per minute $(0.19$ to $0.44 \mathrm{~Hz})$ in this study. Correlation coefficients between the raw $\mathrm{A}_{\text {ref }}$ and the $a c c_{z}$ signals for each inspiratory load level are reported in Table 1. In general, a very strong correlation (from 0.80 to 0.97 ) was observed across the evaluated inspiratory loads. Moreover, a very strong linear correlation $(R=0.98)$ was obtained by comparing the RR values derived from the $\mathrm{A}_{\text {ref }}$ and the $a c c_{z}$ signals as shown in Fig. 3a. Also, the Bland-Altman statistics demonstrated good limits of agreement between the pairs of $R_{\mathrm{Z}}$ and $\mathrm{RR}_{\mathrm{F}}$ values, ranging from -1.44 to 1.46 breaths per minute with a mean bias of 0.01 breaths per minute, as depicted in Fig. 3b. On the other hand, the estimation accuracy, measured in terms of the RMSE in the derived pairs of RR presented a low median error (from 0.13 to 0.28 breaths per minute) and the interquartile range of the RMSE was higher with the increase of the inspiratory load from the quiet breathing to the $25 \mathrm{~cm} \mathrm{H}_{2} \mathrm{O}$ of inspiratory load, as observed in Fig. 4.

\section{DiscUSSION}

In this study, we evaluated the use of the built-in triaxial accelerometer of a smartphone placed on the chest wall for recording the breathing activity and deriving the RR during an inspiratory load respiratory protocol. Gupta and Dantu proposed the use of the accelerometer inside the cell phones to evaluate the patient's breathing in emergency situations [4]. They evaluated the use of the cellphone in vertical and

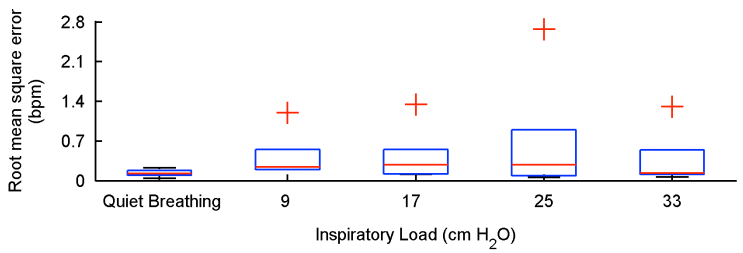

Figure 4. Box plot of the root mean square error between $R_{F}$ and $R R_{Z}$ at different levels of inspiratory load. bpm: breaths per minute. 
horizontal orientation at different locations on the torso to determine the best acceleration component that can be used to measure the RR [4]. In the same line, Pechprasarn and Pongnumkul monitored the RR from a smartphone placed on the chest of a patient lying down [5]. They applied and algorithm based on smoothing and detrending the recorded signals and then estimating the RR by finding the highest peak of the power spectrum. In the present work, the chosen anatomical region to place the smartphone was the right chest, based on a previous study that used an uniaxial accelerometer [10]. Among the three acceleration components of the smartphone we selected the $a c c_{z}$ signal as it gives the most representative information of the chest excursion. However, we also observed some residual respiratory components in the $a c c_{x}$ and the $a c c_{y}$ acceleration signals. This suggests that more information can be extracted using more than one acceleration axis. Moreover, we evaluated the raw signals collected from the smartphone to derive the RR in healthy subjects. As a denoising technique, we applied the EEMD, a noise data assisted algorithm for processing nonlinear and nonstationary signals, as in the case of breathing. EEMD breaks down a signal into IMFs and makes no prior assumption on it. Recently, Mert and Akan have demonstrated that the use of DFA thresholded EMD based denoising (DFA-EMD) method is a powerful tool to separate those oscillatory components that are noisy [13]. They tested the DFA-EMD in synthetic and real epileptic electroencephalographic signals. We extended the use of this technique to the EEMD as the EMD is prone to the mode mixing effect. The $\alpha$ scaling component from DFA method was employed as a threshold to distinguish between noisy and not noisy IMFs and then to reconstruct the denoised signal.

As expected, the use of DFA-EEMD provided an alternative to reduce the inherent noise from the collected raw smartphone accelerometer signals. However, the quality of the recorded signals requires more research in other settings outside the lab as in movement conditions as well as the effect of other noise that can be generated. On the other hand, to validate the use of the smartphone, we compared its recordings with the signals from the reference accelerometer attached over the smartphone. This comparison resulted in a higher correlation, which indicates how well the signals correlated with each other and thus ensured that the smartphone collects adequately the excursion of the chest. A strong correlation and a good agreement were found between the RR derived from the airflow signal (our gold standard) and the accelerometer signal. Furthermore, the $94.67 \%$ of the reported RR were inside the Bland-Altman limits of agreement. We have also observed that the interquartile range of the RMSE was low but it increased with the inspiratory load, from the quiet breathing to the 25 $\mathrm{cm} \mathrm{H}_{2} \mathrm{O}$. We argued that this occurs because the higher the inspiratory load is, the higher is the challenge to maintain the breathing and in consequence the RR detection can be more difficult.

\section{CONCLUSION}

Our preliminary findings suggest the use of the smartphones as potential tools for recording the breathing movement using the built-in acceleration sensors. The use of the smartphone accelerometer signal allows deriving the respiratory rate. Contact and non-contact devices with the patients are being used currently but their use is limited by high costs. The use of mobile devices can become a promising technology for the non-invasively monitoring of physiological data with the associate benefits of being an accessible product of large scale consumption and an easyof-use. More studies will be needed to evaluate the breathing at different patterns, conditions and compare them across male and female populations.

\section{REFERENCES}

[1] World Health Organization, "mHealth: New Horizons for Health Through Mobile Technologies: Second Global Survey on eHealth, Volume 3," Geneva, Switzerland, 2011.

[2] G. Chiarini, P. Ray, S. Akter, C. Masella, and A. Ganz, "mHealth technologies for chronic diseases and elders : a systematic review," IEEE J. Sel. Areas Commun., vol. 31, no. 9, pp. 6-18, Sep. 2013

[3] M. A. Cretikos, R. Bellomo, K. Hillman, J. Chen, S. Finfer, et al., "Respiratory rate: the neglected vital sign," Med. J. Aust., vol. 188, no. 11, pp. 657-659, Jun. 2008

[4] N. K. Gupta and R. Dantu, "Evaluation of respiration quality using smart phone," Proc. 6th Int. Conf. PErvasive Technol. Relat. to Assist. Environ. ACM, May 2013.

[5] T. Pechprasarn and S. Pongnumkul, "Estimation of respiratory rate from smartphone's acceleration data," in 10th Int Conf Electr Eng Comput Telecommun Inf Technol (ECTI-CON) 2013, 2013, pp. 1-5.

[6] F. Q. Al-Khalidi, R. Saatchi, D. Burke, H. Elphick, and S. Tan, "Respiration rate monitoring methods: a review.," Pediatr. Pulmonol., vol. 46, no. 6, pp. 523-529, Jun. 2011.

[7] A. M. Chan, N. Ferdosi, and R. Narasimhan, "Ambulatory respiratory rate detection using ECG and a triaxial accelerometer," in Proc 35th Annu Int Conf IEEE EMBS, 2013, vol. 79, pp. 4058-4061.

[8] A. Fiv, "Android Application: AndroSensor. Google Play Store," 2015. [Online]. Available:

https://play.google.com/store/apps/details?id=com.fivasim.androsenso r. [Accessed: 26-Jan-2015].

[9] N. E. Huang, Z. Shen, S. R. Long, M. C. Wu, H. H. Shih, et al., "The empirical mode decomposition and the Hilbert spectrum for nonlinear and non-stationary time series analysis," Proc. R. Soc. A Math. Phys. Eng. Sci., vol. 454, no. 1971, pp. 903-995, Mar. 1998.

[10] L. Estrada, A. Torres, L. Sarlabous, J. A. Fiz, and R. Jané, "Respiratory rate detection by empirical mode decomposition method applied to diaphragm mechanomyographic signals," in Proc 36th Annu Int Conf IEEE EMBS, 2014, pp. 3204-3207.

[11] Z. Wu and N. E. Huang, "Ensemble empirical mode decomposition: a noise-assisted data analysis method," Advances in Adaptive Data Analysis, vol. 1. pp. 1-41, 2009.

[12] G. Rilling, P. Flandrin, and P. Gonçalvés, "On empirical mode decomposition and its algorithms," in Proceedings of the EURASIP Workshop Nonlinear Signal Image Processing (NSIP), 2003, pp. 8 11.

[13] A. Mert and A. Akan, "Detrended fluctuation thresholding for empirical mode decomposition based denoising," Digit. Signal Process., vol. 32, pp. 48-56, 2014.

[14] C. K. Peng, S. V. Buldyrev, S. Havlin, M. Simons, H. E. Stanley, et al., "Mosaic organization of DNA nucleotides," Phys. Rev. E, vol. 49, no. 2, pp. 1685-1689, 1994. 